Published in final edited form as:

Curr Opin Obstet Gynecol. 2012 October ; 24(5): 299-304. doi:10.1097/GCO.0b013e3283577e9d.

\title{
Expedited partner therapy for sexually transmitted infections
}

\author{
Matthew Hogben ${ }^{a}$, Sarah Kidd ${ }^{a}$, and Gale R. Burstein ${ }^{b}$ \\ aDivision of STD Prevention, Centers for Disease Control and Prevention, Atlanta, Georgia \\ bErie County Department of Health, University at Buffalo, State University of New York, Buffalo, \\ New York, USA
}

\begin{abstract}
Purpose of review-In 2006, the Centers for Disease Control and Prevention recommended Expedited Partner Therapy (EPT) as a clinical option for assuring treatment of sex partners of persons infected with sexually transmitted infections. In this review, we provide an update on research, evaluation and efforts to increase EPT coverage. We also attend to EPT for gonorrhea in the context of antimicrobial resistance.
\end{abstract}

Recent findings-Controlled trials in the United States and United Kingdom have presented increasing variety in intervention approaches. Trials and program evaluations typically demonstrate increased partner treatment rates, although only some studies show reductions in follow-up infection rates. Coverage has increased substantially, with over 30 states permitting EPT for chlamydial infection, gonorrhea, or both. The prospect of cephalosporin-resistant gonorrhea, however, raises the prospect that EPT may become less feasible as a partner treatment approach for gonorrhea patients.

Summary-Clinicians should continue to be aware of the importance of partner managements for STD-infected patients, with EPT being an evidence-based intervention in that respect. The variety in EPT models provides alternatives that may suit some practices and venues. For clinicians seeing gonorrhea patients, effective counseling models - enhanced patient referral should be closely examined in case oral treatment for gonorrhea becomes infeasible.

\section{Keywords}

antimicrobial resistance; partner treatment; sexually transmitted disease/infection; STD prevention programs

\section{INTRODUCTION}

Expedited partner therapy (EPT) is the clinical practice of treating sex partners of persons infected with specific sexually transmitted infections (STI) without requiring an intervening

Correspondence to Matthew Hogben, Centers for Disease Control and Prevention, Mail Stop E-44, Atlanta, GA 30333, USA. Tel: +1 404639 1833; fax: +1 404639 8622; mhogben@cdc.gov.

Conflicts of interest

The findings and conclusions in this report are those of the authors and do not necessarily represent the official position of the US Centers for Disease Control and Prevention.

M.H., S.K. and G.R.B. declare they have no conflicts of interest. 
clinical evaluation [1]. EPT has existed informally for decades, but formal studies and subsequent recommendations for practice in the United States are more recent.

Given an infected index patient in a clinical setting, EPT is based around three core principles: an efficacious oral therapy for treatment, a qualified source of therapy for partner treatment, and a medium through which such therapy can be brought to the partner. In the United States, the relevant STI are uncomplicated chlamydial infection and gonorrhea. The source is a physician or other healthcare provider qualified to prescribe or disburse the single-dose medication(s) for each infection, assuring that both are treated according to current guidelines. The medium is usually the patient, although some health departments allow disease investigators in the field to provide therapy [2]. Given the typical venue for chlamydial infection and gonorrhea diagnosis - a clinic without a specific STI focus or public health field investigation services, patient-delivered partner therapy is the most likely form of EPT and the focus of this review.

\section{RECENT EVIDENCE}

Randomized controlled trials (RCTs) supporting EPT efficacy have been summarized previously [3]. In brief, RCTs in several settings (e.g., STD clinics, family planning clinics) and using various algorithms (e.g., one or two eligible STI, women only or both sexes, availability of public health investigators) have demonstrated that EPT increases the proportion of partners treated and reduces reinfections among index patients at follow-up [4-7]. Subsequent recommendations, published in 2006, were that clinicians consider EPT for heterosexual men and women with uncomplicated chlamydial infection or gonorrhea $[1,8]$. The recommendations express more caution for other infections, as well as for MSM (whose rates of undiagnosed HIV were thought to make them priority candidates for public health investigation services).

Two British studies since 2006 addressed versions of EPT. In Scotland, a RCT with family planning, genitourinary medicine, and hospital termination of pregnancy clinics provided chlamydia-infected women with either medications for partners (azithromycin $1 \mathrm{~g}$ ), test kits for partners to mail back, or patient referral instructions [9]. Data from women reached at 6 months indicated high partner notification rates: across study arms, $86 \%$ of the women said they were able to notify all partners; $97 \%$ were able to notify all or some. Of the $65 \%$ of 330 women enrolled in the study who provided a follow-up sample at some point over the ensuing 21 months, $15 \%$ had at least one positive chlamydia test; positivity rates did not differ across RCT arms.

After EPT with no intervening partner contact with the healthcare system was adjudged legally untenable in England and Wales [10], a UK study assessed treatment rates using two different 'accelerated partner therapy' (APT) models in conjunction with standard partner referral (during which patients received an interview and contact slips) [11"']. In one APT model, patients delivered telephone contact information to partners, who could then be assessed by phone and receive a prescription for care. The other model used community pharmacists trained in sexual health: the index patient was provided with materials for partners to take to the pharmacy, and partners were treated after consultation with the 
pharmacist. Patients were permitted to choose the approach for each of their partners. The 226 patients enrolled reported 296 contactable partners, choosing routine partner notification for $39 \%$, the phone version of APT for $46 \%$, and the pharmacy version for $15 \%$. Both APT methods resulted in higher treatment rates (59\% phone, 66\% pharmacy) than routine partner referral (36\%). Providers preferred the phone version of APT [12"]. In both trials, EPT use was cheaper than alternatives with equivalent efficacy, a point also made when formal cost analyses were applied to earlier RCTs [13"']

Patient choice is an interesting addition to this study. A pure RCT could have shown one condition was superior, and if one mechanism were shown to yield clearly superior treatment rates, a program might not offer the alternative. This approach, however, can easily result in underestimates of effectiveness (e.g., if a participant is randomized to a condition he or she would not have chosen and cannot fulfill). The study authors recommended a clusterrandomized trial, which would permit comparisons of treatment rates in a randomized setting to those in this nonrandomized study.

A RCT in Alabama used trichomoniasis among women as the infection of interest and compared EPT with public health investigator services as well as partner referral [14]. Reinfection rates in the EPT arm were lower than in the other two arms combined, although not lower than in the partner referral arm alone. Relative treatment rates are difficult to interpret, although the differences in treatment estimates were large. For EPT, 80\% of women said they delivered treatment to their male partner, and $90 \%$ of these women thought it very likely the man took the medication. For the other two groups, $25 \%$ of partners in the partner referral condition and $57 \%$ of those in the investigator condition were treated in clinic.

Finally, several program evaluations have also addressed treatment and reinfection in programs instituting EPT. Partner treatment rates for EPT are typically higher than for partner referral, as shown in a study of California family planning clinics [15"']. This same study also demonstrated how EPT could be combined with other partner treatment interventions: asking women receiving test results to bring in partners with them also increased treatment rates, especially for steady partners. Reinfection is harder to measure because patients may be more likely to reappear at a clinic, if they are infected. A New York study in family planning clinics recorded reinfection data for $40 \%$ of patients and saw no difference in reinfection, whereas a Baltimore program measured reinfection at STD clinics and saw it diminish with the implementation of EPT [16,17]. In a San Francisco STD clinic, researchers estimated no differences between the proportion of reinfections in the population receiving and not receiving EPT [18].

\section{COVERAGE AND UPTAKE}

Even an efficacious intervention will have minimal population prevention impact if it is not widely used. The principle of giving patients medications or even prescriptions to pass on to unevaluated partners requires careful attention to issues of safety, legality and liability. 


\section{Legal status}

Following the publication of the 2006 recommendations, the Centers for Disease Control and Prevention (CDC) assessed its legal status of EPT across the United States (note the assessment is explicitly not legal advice), including a review of state laws, legal rulings, and policy statements from organizations such as medical boards, nursing boards, and pharmacy boards [19]. CDC monitors changes: www.cdc.gov/std/ept/legal/default.htm. States are categorized into three areas: those where EPT is permitted, those where EPT is prohibited, and those where EPT is 'potentially allowable' (i.e., states where an authoritative statement would clarify EPT status one way or the other). In 2008, the majority of states fell into the potentially allowable category. Of those where EPT was permitted, some had legislation or other action expressly permitting EPT, whereas others simply had language in state public health law favoring EPT without explicit intent to enable the practice. Since 2008, many states have taken up authorizing legislation or administrative action. At present, 31 states fall into the category of 'permitted,' including six states that originally had prohibitions. Two more are in the final stages of expressly authorizing EPT. Anecdotally, the largest barrier to authorization of EPT is legislative time. A CDC toolkit now helps assure the right stakeholders are consulted: http://www.cdc.gov/std/ept/legal/LegalToolkit.htm.

\section{Uptake}

Data suggest a majority of patients find EPT acceptable [20,21"]. Some patients are averse, however, reinforcing the need for providers to discuss options with patients. Providers appear more ambivalent than patients toward EPT, both in US and UK studies [22,23]. Reasons for ambivalence vary, but often include worries about allergic reactions, liability, and incomplete care when partners do not seek evaluation. One survey of obstetricians and gynecologists in Arizona found that almost one-third of respondents were reluctant to prescribe EPT because of allergy-related concerns, whereas another $11 \%$ did not prescribe EPT due to liability concerns [24]. A New York study reported that health department acceptance was inversely related to the availability of other resources, such as field investigation capacity [25].

None of the US RCTs recorded a serious adverse event, such as anaphylaxis; neither have any of the jurisdictions operating hotlines or other surveil-lance (e.g., California, Washington, Baltimore). As the legal analysis, there have been no records of lawsuits, and several states formally authorizing EPT have built immunity stipulations into their efforts, essentially identifying a standard of care. Insurance, however, may not cover medications for partners, which means either the clinician, health organization, or the patient and partner have to cover the cost of medication.

\section{Uptake evaluation}

In New York City, a family planning clinic instituted EPT as part of a partner management strategy, with providers trained to 'address partner treatment in the context of a suspected or confirmed case of chlamydia,' in particular, through a discussion with the patient [16]. Of 466 patients treated for chlamydia in 2004-2005, 323 (69\%) were provided with EPT after a discussion with their provider. Of the remainder, $36(8 \%)$ had no partner treatment information recorded, 58 (12\%) chose partner referral only, 31 (7\%) chose 'nothing,' and 18 
(4\%) reported their partners were already treated, rendering them ineligible for EPT. Baltimore STD clinics [17] have reported substantial proportions of individuals receiving EPT (68\%), as has an STD clinic in San Francisco (43\%, with almost identical rates of uptake among heterosexual patients and MSM)[18]. Each of these locations is characterized by clear protocols and a sense that partner treatment is an important part of patient and public health. The Arizona survey cited above also found that providers who were informed of guidelines permitting EPT were more likely to prescribe EPT, 69 vs. 44\% [24].

Uptake may be improved by adding EPT options to other innovations, especially if those innovations address barriers such as reluctance to prescribe without some type of partner contact. For example, internet-based partner services (IPS), in which disease investigators or patients contact partners through electronic media, help link partners to health services. Although the typical construal of IPS is to contact partners and bring them to in-person care, a conceivable extension for uncomplicated chlamydial infection would be to gather some basic partner data, answer questions and prescribe accordingly - similar to APT. Electronic contact may be proactive, as when an investigator attempts to contact a partner, or reactive, as when a patient is given a link or e-mail to contact health services. Although a proactive approach is likely to be more efficacious, a reactive approach may be easier to implement, especially on a population-wide basis.

\section{EXPEDITED PARTNER THERAPY AND ANTIMICROBIAL RESISTANCE}

EPT for gonorrhea is complicated by the emerging problem of antimicrobial-resistant Neisseria gonorrhoeae. N. gonorrhoeae has progressively acquired resistance to each antibiotic previously recommended for treatment: sulfonamides, penicillin, tetracycline, and, most recently, fluoroquinolones. Now, there is evidence that $N$. gonorrhoeae is becoming less susceptible to oral third generation cephalosporins. Cephalosporins are the last class of antibiotics that remain widely effective and are recommended for the treatment of gonorrhea. In the last decade, decreased in-vitro susceptibility to cephalosporins has been observed in Asia, Europe, and North America [26-31] and oral cefixime treatment failures have been reported from Japan and several countries in Western Europe [32-38]. In the United States' Gonococcal Isolate Surveillance Project (GISP), cefixime minimum inhibitory concentrations (MICs) against $N$. gonorrhoeae have been increasing, indicating declining susceptibility to cefixime. Although there have been no confirmed cephalosporin treatment failures in the United States, from 2006 to January-August 2011, the proportion of GISP isolates with elevated cefixime MICs ( $\searrow 0.25 \mu \mathrm{g} / \mathrm{ml}$ ) increased from 0.1 to $1.5 \%$ [39"']. (The MIC breakpoints corresponding to cephalosporin resistance have not been defined, but the Clinical and Laboratory Standards Institute defines 'decreased susceptibility' to cephalosporins as cephalosporin MICs $\searrow 0.5 \mu \mathrm{g} / \mathrm{ml}$. GISP uses the lower MIC breakpoints of $\searrow 0.25 \mu \mathrm{g} / \mathrm{ml}$ for cefixime and $\searrow 0.125 \mu \mathrm{g} / \mathrm{ml}$ for ceftriaxone, considered 'elevated MICs', to provide greater sensitivity for surveillance trends). In the West, the proportion increased from $0.2 \%$ in 2006 to $3.2 \%$ during January-August 2011. Among MSM, the proportion increased from 0.2 to $3.8 \%$. The proportion of isolates with elevated ceftriaxone MICs also increased during this time, but was at least $1 \%$ in all regions and among MSM during January-August 2011. 
Due to these trends in decreasing susceptibility to cefixime, CDC recently revised its gonorrhea treatment guidelines and no longer recommends cefixime as first-line treatment for gonorrhea [39"']. The CDC-recommended gonorrhea treatment regimen is dual treatment with ceftriaxone $250 \mathrm{mg}$ given intramuscularly as a single dose and either oral azithromycin $1 \mathrm{~g}$ as a single dose or oral doxycycline $100 \mathrm{mg}$ twice a day for 7 days. For patients with severe cephalosporin allergy, oral azithromycin $2 \mathrm{~g}$ as a single dose is the only alternative. However, this regimen is not recommended for routine treatment because $N$. gonorrhoeae has previously rapidly developed resistance to macrolide antibiotics, and cases of high-level azithromycin resistance have already been documented $\left[40^{-}, 41\right]$. Oral cefixime $400 \mathrm{mg}$ as a single dose, in combination with either azithromycin or doxycycline, should be used only when treatment with ceftriaxone is not possible. CDC recommends that all patients who receive an alternative treatment regimen return for test of cure 1 week after treatment to ensure that the infection has cleared.

Because the only CDC-recommended treatment regimen includes an intramuscular injection, the revised treatment guidelines have significant implications for EPT for gonorrhea. Patients and their exposed sex partners should be informed that dual therapy with ceftriaxone and either azithromycin or doxycycline is the most effective treatment for gonorrhea, and partners should be strongly advised to present to a clinic for dual treatment that includes ceftriaxone. However, for heterosexual patients with gonorrhea whose partners' treatment with ceftriaxone cannot be ensured or is unlikely, EPT using cefixime and either azithromycin or doxycycline can still be considered. This approach should always be accompanied by efforts to encourage partners to seek clinical evaluation and to educate partners about the need for test of cure if a cefixime-based regimen is used. In the future, if and when cefixime resistance emerges in the United States, continued use of cefixime-based EPT for treatment of gonorrhea will require ongoing risk-benefit analyses that take into account the prevalence of cefixime-resistant strains in the community. New oral treatment regimens with a high efficacy against $N$. gonorrhoeae are urgently needed; until new oral regimens are developed, cephalosporin resistance poses a threat to the continued use of EPT for gonorrhea.

\section{CONCLUSION}

EPT for chlamydial infection is increasingly available as an option and relatively uncontroversial. The authors of one RCT [7] noted that patients had received 'gold standard' counseling and suggested EPT could be most useful in situations where such counseling was not likely to occur. Another reasonable deduction from these data, however, is that providers and organizations actually have two routes to increasing the success of patient-based partner notification: EPT is one, and improved counseling is another [6,42]. This conclusion may become especially salient in gonorrhea treatment. The current first-line recommendation for gonor-rhea treatment requires an injection; therefore, effective partner referral counseling assumes a greater relative primacy for gonorrhea. Once again, situational and patient-level contingencies may well determine the best course of action in various settings, and a frank and informed discussion between clinician and patient remains as important as ever. 


\section{REFERENCES AND RECOMMENDED READING}

Papers of particular interest, published within the annual period of review, have been highlighted as:

- of special interest

- of outstanding interest

Additional references related to this topic can also be found in the Current World Literature section in this issue (p. 356).

1. Centers for Disease Control and Prevention. Expedited partner therapy in the management of sexually transmitted diseases: Review and guidance. Atlanta, GA: Department of Health and Human Services, 2006.

2. Steiner KC, Davila V, Kent CK, et al. Field-delivered therapy increases treatment for chlamydia and gonorrhea. Am J Public Health 2003; 93:882-884. [PubMed: 12773344]

3. Kissinger P, Hogben M. Expedited partner therapy for sexually transmitted infections: an update. Curr Infect Dis Rep 2011; 13:188-195. [PubMed: 21365383]

4. Schillinger JA, Kissinger P, Calvet H, et al. Patient-delivered partner treatment with azithromycin to prevent repeated Chlamydia trachomatis infection among women. Sex Transm Dis 2003; 30:49-56. [PubMed: 12514443]

5. Golden MR, Whittington WW, Handsfield HH, et al. Impact of expedited sex partner treatment on recurrent or persistent gonorrhea or chlamydial infection: a randomized controlled trial. New Engl J Med 2005; 352:676-685. [PubMed: 15716561]

6. Kissinger P, Mohammed H, Richardson-Alston G, et al. Patient-delivered partner treatment for male urethritis: a randomized controlled trial. Clin Infect Dis 2005; 41:623-629. [PubMed: 16080084]

7. Trelle S, Shang A, Nartey L, et al. Improved effectiveness of partner notification for patients with sexually transmitted infections: Systematic review. BMJ 2007; 334:354. [PubMed: 17237298]

8. Centers for Disease Control and Prevention. Sexually Transmitted Disease treatment guidelines, 2010. MMWR 2010; 59:1-110.

9. Cameron ST, Glasier A, Scott G, et al. Novel interventions to reduce re-infection in women with chlamydia: a randomized controlled trial. Hum Reprod 2009; 24:888-895. [PubMed: 19136481]

10. Golden MR, Estcourt CS. Barriers to the implementation of expedited partner therapy. Sex Transm Inf 2011; 87:i37-i38.

11. Estcourt C, Sutcliffe L, Cassell J, et al. Can we improve partner notification rates through expedited partner therapy in the UK? Findings from an exploratory trial of Accelerated Partner Therapy (APT). Sex Transm Inf 2012; 88:21-26. - This UK nonrandomized trial of accelerated partner therapy presents two new models of expediting partner treatment, while retaining pretreatment contact with the healthcare system (pharmacists or telephone consultations).

12. Shackleton T, Sutcliffe L, Estcourt C. Is Accelerated Partner Therapy partner notification for sexually transmissible infections acceptable and feasible in general practice? Sex Health 2011; 8:17-22. [PubMed: 21371378] - Drawing from UK nonrandomized study data, this analysis provides recent estimates and nuance around provider and patient preferences with respect to expediting partner treatment.

13. Gift TL, Kissinger P, Mohammed H, et al. The cost and cost-effectiveness of expedited partner therapy compared with standard partner referral for the treatment of chlamydia or gonorrhea. Sex Transm Dis 2011; 38:1067-1073. [PubMed: 21992986] - This article addresses frequently asked questions around the cost-effectiveness of EPT, in particular compared with other patient-based referral methods.

14. Schwebke JR, Desmond RA. A randomized controlled trial of partner notification methods for prevention of trichomoniasis in women. Sex Transm Dis 2010; 37:392-396. [PubMed: 20453720]

15. Yu YY, Frasure-Williams JA, Dunne EF, et al. Chlamydia partner services for females in California family planning clinics. Sex Transm Dis 2011; 38:913-918. [PubMed: 21934563] $\boldsymbol{m}$ This analysis conducted from family planning clinic data, which could be widely available, shows how EPT 
works in routine clinical practice, but also how EPT can be used in conjunction with other brief and feasible interventions.

16. Kerns JL, Jones HE, Pressman EJ, et al. Implementation of expedited partner therapy among women with chlamydia infection at an urban family planning clinic. Sex Transm Dis 2011; 38:722-726. [PubMed: 21844724]

17. Ames AK, Muvva R, Miazad R, et al. Expedited partner therapy for gonorrhea and chlamydia decreases visits for reinfection among STD clinic patients. Atlanta, GA: National STD Prevention Conference; 2010.

18. Stephens SC, Bernstein KT, Katz MH, et al. The effectiveness of patient-delivered partner therapy and chlamydial and gonococcal reinfection in San Francisco. Sex Transm Dis 2010; 37:525-529. [PubMed: 20502392]

19. Hodge JG, Pulver A, Hogben M, et al. Expedited partner therapy for sexually transmitted diseases: Assessing the legal environment. Am J Public Health 2008; 98:238-243. [PubMed: 18172137]

20. Melvin L, Cameron ST, Glasier A, et al. Preferred strategies of men and women for managing chlamydial infection. Brit J Obstet Gyn 2009; 116:357-365.

21. Gursahaney PR, Jeong K, Dixon BW, Wiesenfeld HC. Partner notification of sexually transmitted diseases: Practices and preferences. Sex Transm Dis 2011; 38:821-827. [PubMed: 21844737] - A rarely-tested point in this study is the distinction between what patients are willing to do with respect to partner management and what their first preferences are.

22. McNutt LA, Davis CF, Bednarczyk RA, et al. Alternative approaches to partner notification, diagnosis, and treatment: Pharmacists' perspectives on proposed patient delivered partner therapy in New York State, 2007. Sex Transm Dis 2009; 36:178-184. [PubMed: 19174732]

23. Shivasankar S, Challenor R. Patient-delivered partner therapy in the UK: what do the professionals think? Int J STD AIDS 2008; 19:437-440. [PubMed: 18574112]

24. Taylor MM, Collier MG, Winscott MM, et al. Reticence to prescribe: utilization of expedited partner therapy among obstetrics providers in Arizona. Int J STD AIDS 2011; 22:449-452. [PubMed: 21742811]

25. Davis CF, Cummings M, Coles BF, McNutt LA. Alternative approaches to partner notification, diagnosis, and treatment: Perspectives of New York County health departments, 2007. Sex Transm Dis 2009; 36:185-190. [PubMed: 19174730]

26. Ito M, Yasuda M, Yokoi S, et al. Remarkable increase in central Japan in 2001-2002 of Neisseria gonorrhoeae isolates with decreased susceptibility to penicillin, tetracycline, oral cephalosporins, and fluoroquinolones. Antimicrob Agents Chemother 2004; 48:3185-3187. [PubMed: 15273147]

27. Tanaka M, Nakayama H, Tunoe H, et al. A remarkable reduction in the susceptibility of Neisseria gonorrhoeae isolates to cephems and the selection of antibiotic regimens for the single-dose treatment of gonococcal infection in Japan. J Infect Chemother 2002; 8:81-86. [PubMed: 11957125]

28. Su X, Jiang F, Quimuge, et al. Surveillance of antimicrobial susceptibilities in Neisseria gonorrhoeae in Nanjing, China, 1999-2006. Sex Transm Dis 2007; 34:995-999. [PubMed: 17595594]

29. Cole MJ, Chisholm SA, Hoffmann S, et al. European surveillance of antimicrobial resistance in Neisseria gonorrhoeae. Sex Transm Dis 2010; 36:427-432.

30. Martin I, Swatzky P, Allen V, et al. Emergence and characterization of Neisseria gonorrhoeae isolates with decreased susceptibilities to ceftriaxone and cefixime in Canada: 2001-2010. Sex Transm Dis 2012; 39:316-323. [PubMed: 22421701]

31. Centers for Disease Control and Prevention. Cephalosporin susceptibility among Neisseria gonorrhoeae isolates: United States, 2000-2010. MMWR 2011; 60:873-877. [PubMed: 21734634]

32. Deguchi T, Yasuda M, Yokoi S, et al. Treatment of uncomplicated gonococcal urethritis by doubledosing of $200 \mathrm{mg}$ cefixime at a 6-h interval. J Infect Chemother 2003; 9:35-39. [PubMed: 12673405]

33. Yokoi S, Deguchi T, Ozawa T, et al. Threat to cefixime treatment for gonorrhea. Emerg Infect Dis 2007; 13:1275-1277. [PubMed: 17953118]

34. Unemo M, Golparian D, Stary A, Eigentler A. First Neisseria gonorrhoeae strain with resistance to cefixime causing gonorrhoeae treatment failure in Austria, 2011. Eurosurv 2011; 16. 
35. Unemo M, Golparian D, Syversen G, et al. Two cases of verified clinical failures using internationally recommended first-line cefixime for gonorrhoea treatment, Norway, 2010. Eurosurv 2010; 15.

36. Unemo M, Golparian D, Nicholas R, et al. High-level cefixime- and ceftriaxone-resistant N. gonorrhoeae in Europe (France): novel penA mosaic allele in a successful international clone causes treatment failure. Antimicrob Agents Chemother 2012; 56:1273-1280. [PubMed: 22155830]

37. Ison CA, Hussey J, Sankar KN, et al. Gonorrhoea treatment failures to cefixime and azithromycin in England, 2010. Eurosurv 2011; 16.

38. Forsyth S, Penney P, Rooney G. Cefixime-resistant Neisseria gonorrhoeae in the UK: a time to reflect on practice and recommendations. Int J STD AIDS 2011; 22:296-297. [PubMed: 21571983]

39. CDC. Update to CDC's Sexually Transmitted Diseases Treatment Guidelines, 2010: Oral Cephalosporins No Longer a Recommended Treatment forGonococcal Infections. MMWR 2012; 61:590-594. [PubMed: 22874837] $\boldsymbol{m}$ Informed commentary and revised guidance on gonorrhea management, with significant implications for partner treatment, including EPT.

40. Katz AR, Komeya AY, Soge OO, et al. Neisseria gonorrhoeae with high-level resistance to azithromycin: case report of the first isolate identified in the United States. Clin Infect Dis 2012; 54:841-843. [PubMed: 22184617] - An account of gonorrhea resistance to azithromycin treatment, part of the current dual treatment regimen for gonorrhea.

41. Chisholm SA, Neal TJ, Alawattegama AB, et al. Emergence of high-level azithromycin resistance in Neisseria gonorrhoeae in England and Wales. J Antimicrob Chemother 2009; 64:353-358. [PubMed: 19468025]

42. Wilson TE, Hogben M, Malka E, et al. Reducing sexually transmitted infection rates by improving patient referral for treatment: a randomized, controlled trial. Am J Public Health 2009; 99 (suppl):S104-S110. [PubMed: 18556619] 


\section{KEY POINTS}

- $\quad$ Treating partners of persons infected with sexually transmitted diseases is important for patients, their partners, and communities.

- $\quad$ EPT is one means of increasing the likelihood that partners will be treated for exposure to chlamydia or gonorrhea.

- $\quad$ Availability of EPT is consistently increasing, although barriers to EPT use still remain.

- The prospect of cephalosporin-resistant gonorrhea may limit EPT for gonorrhea (but not chlamydia). 